\title{
A case of intrahepatic cholangiocarcinoma that was difficult to diagnose prior to surgery: A case report
}

\author{
KEN NAGATA ${ }^{1}$, TAKAHIRO EINAMA ${ }^{1}$, AKIFUMI KIMURA ${ }^{2}$, MICHINORI MURAYAMA ${ }^{2}$, \\ HIROTERU TAKEO ${ }^{3}$, MAKOTO NISHIKAWA ${ }^{1}$, MAYUMI HOSHIKAWA ${ }^{1}$, TAKUJI NORO ${ }^{1}$, \\ SHO OGATA $^{4}$, SUEFUMI AOSASA ${ }^{1}$, YOSHIKI KAJIWARA ${ }^{1}$, EIJI SHINTO ${ }^{1}$, \\ YOSHIHISA YAGUCHI $^{1}$, SHUICHI HIRAKI ${ }^{1}$, HIRONORI TSUJIMOTO ${ }^{1}$, \\ KAZUO HASE $^{1}$, HIDEKI UENO ${ }^{1}$ and JUNJI YAMAMOTO ${ }^{1}$
}

\author{
${ }^{1}$ Department of Surgery, National Defense Medical College, Tokorozawa, Saitama 359-8513; \\ Departments of ${ }^{2}$ Surgery and ${ }^{3}$ Diagnostic Pathology, Self-Defense Forces Central Hospital, Setagaya, Tokyo 154-8532; \\ ${ }^{4}$ Department of Pathology, National Defense Medical College, Tokorozawa, Saitama 359-0042, Japan
}

Received May 15, 2018; Accepted September 7, 2018

DOI: $10.3892 / \mathrm{ol} .2018 .9666$

\begin{abstract}
The present study reports a case of mass-forming intrahepatic cholangiocarcinoma (ICC), which mimicked cholangiocellular carcinoma (CoCC) during imaging and a needle biopsy examination. A 51-year-old female with no relevant medical history was referred to the National Defense Medical College hospital with an intrahepatic tumor. Computed tomography demonstrated non-homogeneous enhancement in the early arterial phase and persistent enhancement in the portal and equilibrium phases, together with notable swelling of the para-aortic lymph nodes. Gadolinium-ethoxybenzyl diethylenetriamine-pentaacetic acid-enhanced magnetic resonance imaging revealed low signal intensity in the hepatobiliary phase. The liver tumor and lymph nodes exhibited increased radiotracer uptake (maximum standardized uptake value $=14.0$ ) with positron emission tomography. A histological examination of a percutaneous needle biopsy specimen of the liver tumor indicated a diagnosis of CoCC. The patient underwent left hepatectomy and lymphadenectomy. The surgical specimen contained a poorly differentiated adenocarcinoma with anaplastic changes, which was immunohistochemically positive for epithelial membrane antigen (at the luminal membrane), cytokeratins 7 and 19, and negative for $\alpha$-fetoprotein, hepatocyte-specific antigen, cluster of differentiation 56 and KIT. Based on these histopathological and immunohistochemical findings, the patient was diagnosed with ICC.
\end{abstract}

Correspondence to: Dr Takahiro Einama, Department of Surgery, National Defense Medical College, 3-2 Namiki, Tokorozawa, Saitama 359-8513, Japan

E-mail: titiuehahaue@hotmail.com

Key words: intrahepatic cholangiocarcinoma, cholangiocellular carcinoma, diagnosis

\section{Introduction}

Despite notable progress in the ability to preoperatively diagnose hepatic tumors due to recent technological advances in radiological imaging techniques, it may be difficult to obtain a precise preoperative diagnosis for liver tumors $(1,2)$. Intrahepatic cholangiocarcinoma (ICC) has been reported to exhibit various imaging features such as irregular margins, thin peripheral arterial enhancement with progressive centripetal filling and delayed enhancement of the central portions, depending on the tumor's location, size and intratumoral components (3). Additionally, computed topography (CT) findings in cholangiocellular carcinoma (CoCC) have demonstrated the histological features of the tumor (4). Furthermore, enhanced CT indicated the early enhancement and delayed washout of $\mathrm{CoCC}$, similar to hepatocellular carcinoma (HCC) (5), or ringed enhancement at the periphery during the early phase and persistent enhancement during the delayed phase, similar to ICC on enhanced CT (6). Therefore, percutaneous needle biopsy examinations may be performed to diagnose liver tumors preoperatively. However, needle biopsy may yield different results depending on the region of the tumor that is biopsied due to the heterogeneous distribution of cells in liver tumors (7). The present study reports a case of mass-forming ICC, which mimicked CoCC during imaging and needle biopsy examination.

\section{Case report}

A liver tumor was incidentally located in a 51-year-old female during a medical check-up in September 2016. The patient had no significant medical, family, including with regard to genetic information, or psychosocial history of liver tumors. A routine physical examination produced normal results. No peripheral blood-, blood chemistry- or hemostasis-associated abnormalities were determined, except for a slightly elevated serum $\gamma$-glutamyl transpeptidase level of $63 \mathrm{U} / 1$ (normal range, $<50 \mathrm{U} / \mathrm{l})$. The patient's tumor marker levels, including those of carcinoembryonic antigen $[0.7 \mathrm{ng} / \mathrm{ml}$ (normal range, 


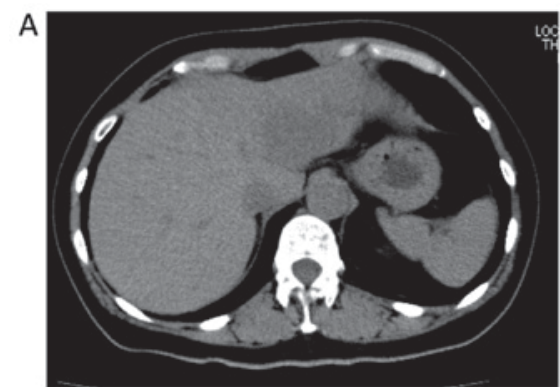

Plain

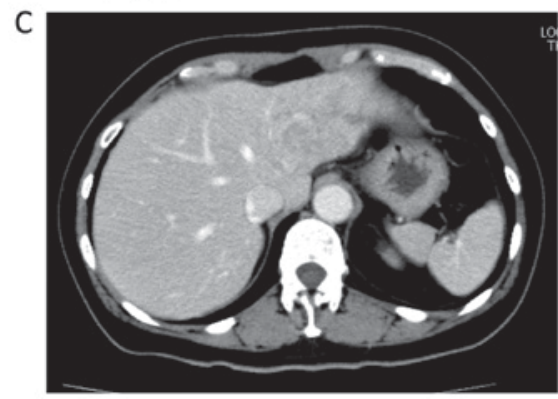

Portal phase

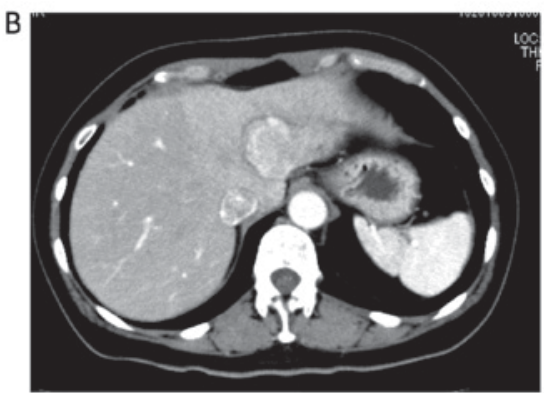

Arterial phase

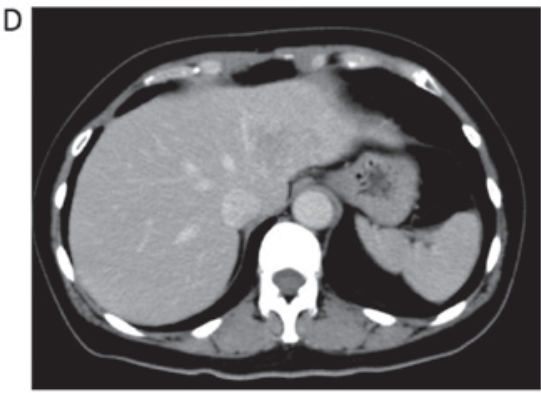

Equilibrium phase

Figure 1. Preoperative abdominal CT of the liver tumor. (A) CT demonstrated a low-density mass, measuring 50 mm in the largest diameter, in segment 2 of the liver. (B) The tumor exhibited slight enhancement during the arterial phase and persistent enhancement during the (C) portal and (D) equilibrium phases. CT, computed tomography.

$<5.0 \mathrm{ng} / \mathrm{ml})]$, carbohydrate antigen $19-9[7.5 \mathrm{U} / \mathrm{ml}$ (normal range, $<37 \mathrm{U} / \mathrm{ml}$ )], $\alpha$-fetoprotein $[\mathrm{AFP} ; 4.3 \mathrm{U} / \mathrm{ml}$ (normal range, $<20 \mathrm{U} / \mathrm{ml}$ )], and protein induced by vitamin $\mathrm{K}$ absence or antagonists-II [14 U/ml (normal range, $<40 \mathrm{U} / \mathrm{ml}$ )], were within the normal limits. Abdominal ultrasonography revealed a $50-\mathrm{mm}$ tumor in the lateral segment of the liver. Computed tomography (CT) demonstrated non-homogeneous enhancement in the arterial phase, persistent enhancement in the portal and equilibrium phases (Fig. 1A-D), and swelling and enhancement of the para-aortic lymph nodes. Gadolinium-ethoxybenzyl diethylenetriamine-pentaacetic acid-enhanced magnetic resonance imaging revealed low signal intensity in the hepatobiliary phase (Fig. 2A and B). Positron emission tomography/CT indicated fludeoxyglucose uptake by the liver tumor and lymph nodes (Fig. 3A-C). Due to the absence of underlying liver disease, elevated tumor marker levels and specific imaging findings, ultrasonography-guided percutaneous needle biopsy of the liver tumor was performed.

Histological examination of $10 \%$ formalin-fixed $(24 \mathrm{~h}$, room temperature), 3- $\mu \mathrm{m}$ paraffin-embedded sections was performed using hematoxylin-eosin (HE) and mucin staining. For HE staining, sections were stained with $0.12 \mathrm{~g} / \mathrm{v} \%$ hematoxylin solution produced by Hematoxylin (C.I. 75290) cryst. (cat. no. 104302; Merck KGaA, Darmstadt, Germany) three times ( $3 \mathrm{~min} / \mathrm{stain})$ at room temperature, and with eosin $\mathrm{Y}$ (cat. no. 058-00062; Wako Pure Chemical Industries, Ltd., Osaka, Japan) two times (1.5 min/stain) at room temperature. For mucin staining, sections were stained with Schiff's Reagent (cat. no. 40921; Muto Pure Chemicals Co. Ltd., Tokyo, Japan) for $15 \mathrm{~min}$ at room temperature, and with Alcian Blue Stain Solution pH 2.5 for Histology (cat. no. 40852; Muto Pure Chemicals Co. Ltd.) for $20 \mathrm{~min}$ at room temperature. Staining was detected under a light microscope.
For immunohistochemistry, $10 \%$ formalin-fixed $(24 \mathrm{~h}$, room temperature), $3-\mu \mathrm{m}$ paraffin-embedded sections were analyzed. The following commercially available primary antibodies were used: Anti-cytokeratin (CK)7 (cat. no. M7018; 1:200; Dako; Agilent Technologies, Inc., Santa Clara, CA, USA), CK19 (cat. no. M0888; 1:100; Dako; Agilent Technologies, Inc.), CK20 (cat. no. M7019; 1:100; Dako; Agilent Technologies, Inc.), glypican 3 (cat. no. 718021; Nichirei Bioscience, Inc., Tokyo, Japan), CEA (cat. no. A115; 1:2,000; Dako; Agilent Technologies, Inc.), cluster of differentiation 56 (CD56; cat. no. 713331; Nichirei Bioscience, Inc.), AFP (cat. no. A0008; 1:500; Dako; Agilent Technologies, Inc.), hepatocyte-specific antigen (cat. no. NCL-HSA; 1:50; Novocastra; Leica Microsystems GmbH, Wetzler, Germany), epithelial membrane antigen (EMA; cat. no. M0613; 1:200; Dako; Agilent Technologies, Inc.) and KIT (cat. no. 713391; Nichirei Bioscience, Inc.). Briefly, sections were prepared as follows: For glypican 3 detection, samples underwent heat-induced epitope-retrieval [boiled at $100^{\circ} \mathrm{C}$ for $40 \mathrm{~min}$ in Heat Processor Solution pH 9 (cat. no. 715291; 1:10; Nichirei Bioscience, Inc.)]; for CD56 detection, samples underwent heat-induced epitope retrieval [boiled at $100^{\circ} \mathrm{C}$ for $40 \mathrm{~min}$ in Heat Processor Solution pH 6 (cat. no. 715281; 1:10; Nichirei Bioscience, Inc.)]; for CK7, 19 and 20 detection, samples underwent, proteinase-induced epitope retrieval [protease $\mathrm{K}$ (cat.no. 715231; Nichirei Bioscience, Inc.)] at room temperature for $10 \mathrm{~min})$. After blocking with $3 \mathrm{v} / \mathrm{v} \%$ hydrogen peroxidase solution (cat. no. 715142; Nichirei Bioscience, Inc.) for $5 \mathrm{~min}$ at room temperature, the sections were incubated with primary antibodies for $30 \mathrm{~min}$ at room temperature, followed by incubation with Histofine ${ }^{\circledR}$ Simple Stain MAX PO (MULTI) (cat. no. 724152; Nichirei Bioscience, Inc.) for $30 \mathrm{~min}$, and finally with Histofine ${ }^{\circledR}$ Simple Stain DAB substance kit 
A B

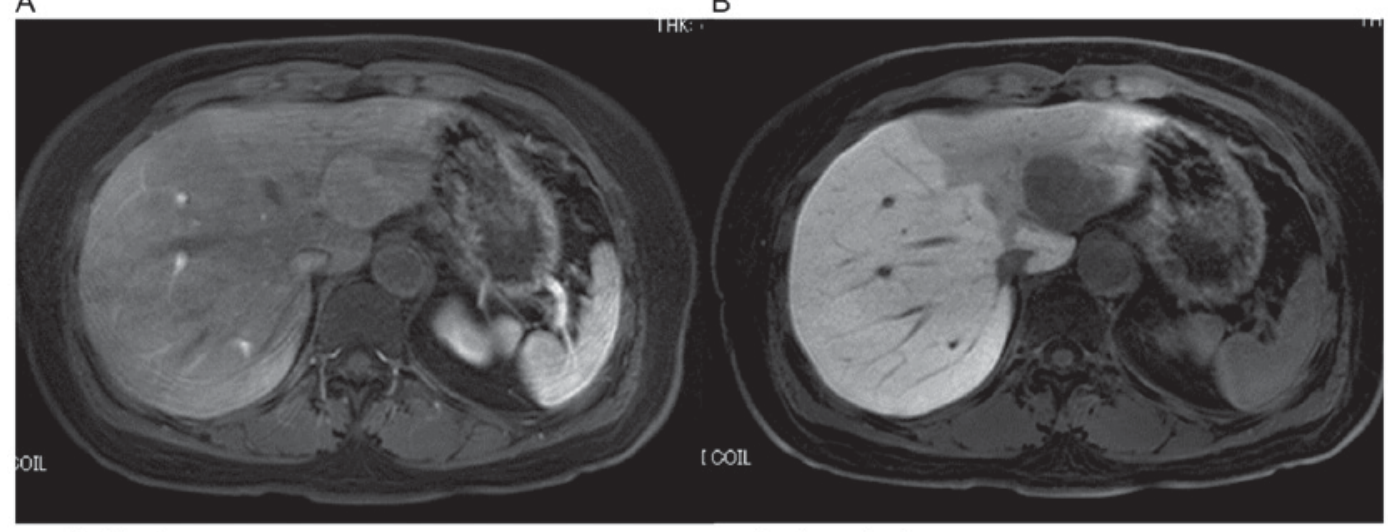

Early phase

Delayed phase

Figure 2. Gd-EOB-DTPA-enhanced MRI of the liver tumor. (A) Gd-EOB-DTPA-enhanced MRI demonstrated mild heterogeneous internal enhancement in the early phase; (B) whereas, certain regions exhibited washout in the delayed phase. Gd-EOB-DTPA-enhanced MRI, gadolinium-ethoxybenzyl diethylenetriamine-pentaacetic acid-enhanced magnetic resonance imaging.

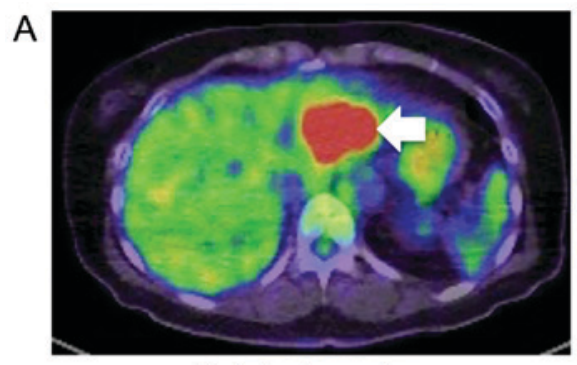

Axial plane-1

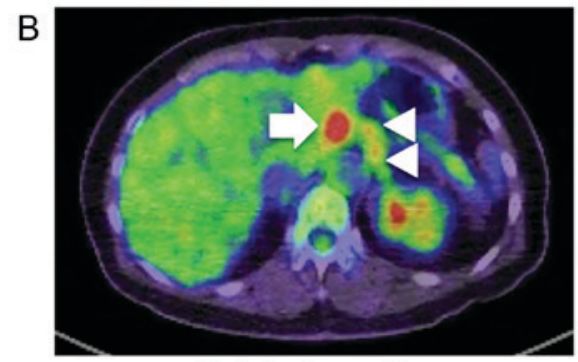

Axial plane-2

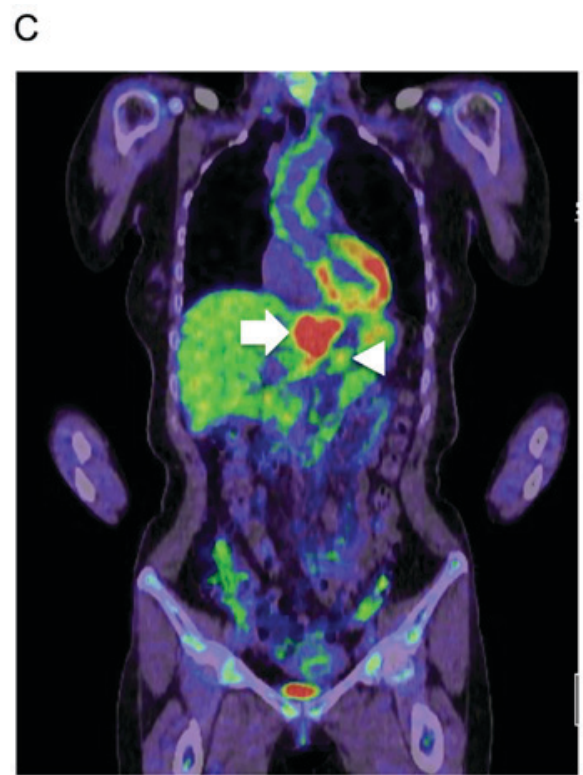

Coronal plane

Figure 3. FDG-PET of the liver tumor. FDG-PET demonstrated FDG-avid lesions in the left hepatic lobe in the (A) axial plane and para-aortic lymph nodes in the (B) axial plane and the (C) coronal plane. No other notable FDG deposits were determined. Arrow, tumor; arrowhead, swollen para-aortic lymph nodes; FDG, fludeoxyglucose; PET, positron emission tomography.

(cat. no. 725191; Nichirei Bioscience, Inc.; 5 min x2 incubations). The steps following incubation with primary antibodies were performed under a HISTOSTAINER 48A autostaining machine (Nichirei Bioscience, Inc.) at room temperature.

A histopathological examination of the biopsy specimen demonstrated spindle-shaped tumor cells with slightly eosinophilic cytoplasm and enlarged nuclei, arranged in a cord-like pattern. An immunohistochemical examination was performed, according to the manufacturers' protocols; the results indicated that the tumor cells were positive for CK7 and glypican 3, partially positive for CD56 and negative for CK20, AFP, and hepatocyte-specific antigen (Fig. 4A-E). EMA staining revealed positivity in the luminal membranes of the tumor cells (Fig. 4F) $(8,9)$. No mucus production was detected with Periodic acid Schiff-alcian blue (pH 2.5) staining. According to these findings, the patient was preoperatively diagnosed with CoCC (cT2N1M0 stage II, according to the eighth edition of the Tumor-Node-Metastasis Classification of Malignant Tumors developed by the Union for International Cancer Control) (10). The patient underwent left hepatectomy and lymphadenectomy 72 days after her first visit to the National Defense Medical College Hospital. The regional lymph nodes, which were identified based on preoperative imaging and an intraoperative assessment, were removed. The regional lymph nodes were located in the hepatic hilum and hepatoduodenal ligament, posterior to the upper portion of the pancreatic head, and at the common hepatic artery and para-aortic stations. The operative time was $510 \mathrm{~min}$, and the total amount of intraoperative blood loss was $721 \mathrm{~g}$. The patient's postoperative recovery was uneventful, and she was discharged from the hospital on postoperative day 15 . 
A

B

C

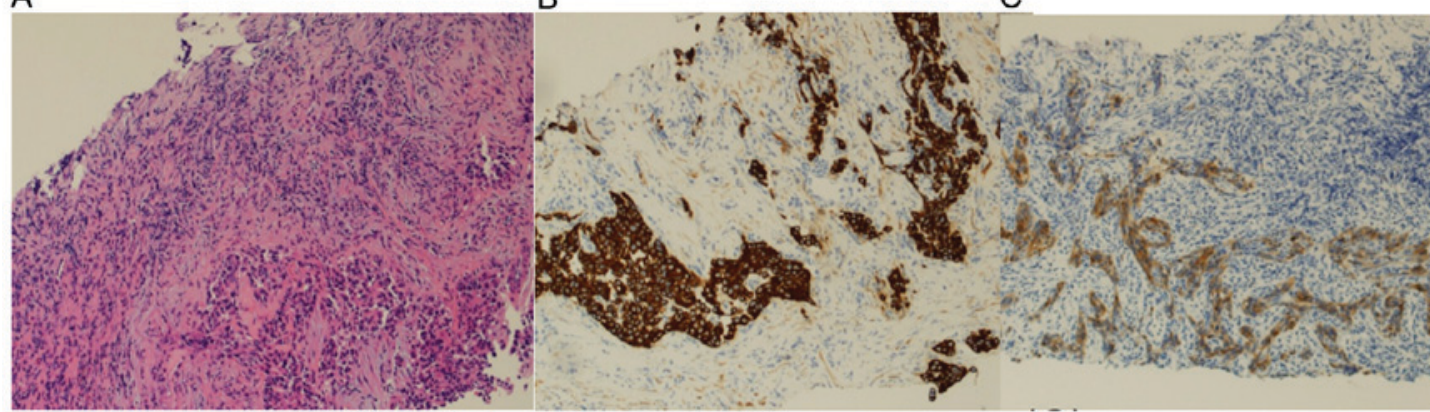

D

E

F

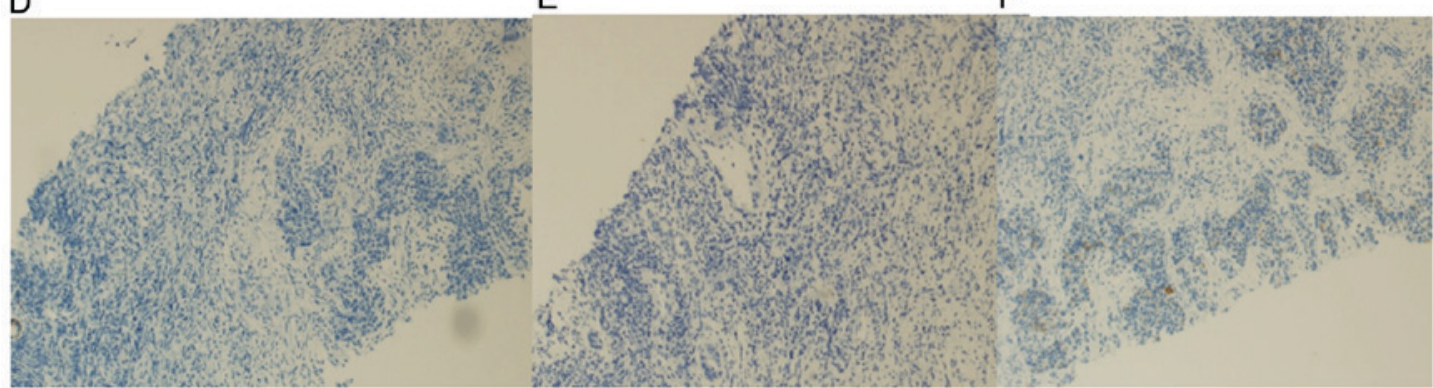

Figure 4. Histopathological analysis of the liver tumor obtained during the needle biopsy. (A) Hematoxylin-eosin staining of the microscopic specimen demonstrated that the tumor cells had slightly eosinophilic cytoplasm and round nuclei with clear nucleoli. Immunohistochemical staining of (B) CK7, (C) EMA, (D) CD56, (E) AFP (F) and glypican 3 demonstrated that the tumor cells were diffusely positive for CK7, positive for EMA (in the luminal membranes), and negative for CD56, AFP and glypican 3 (magnification, x20). CD56, cluster of differentiation; CK, cytokeratin; AFP, $\alpha$-fetoprotein; EMA, epithelial membrane antigen.

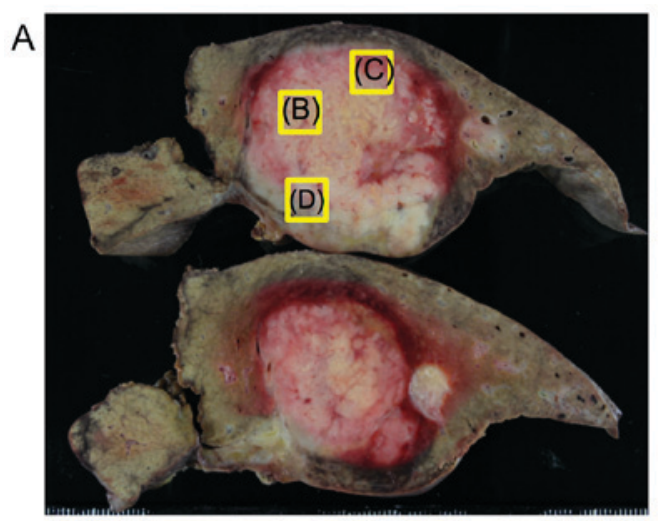

B
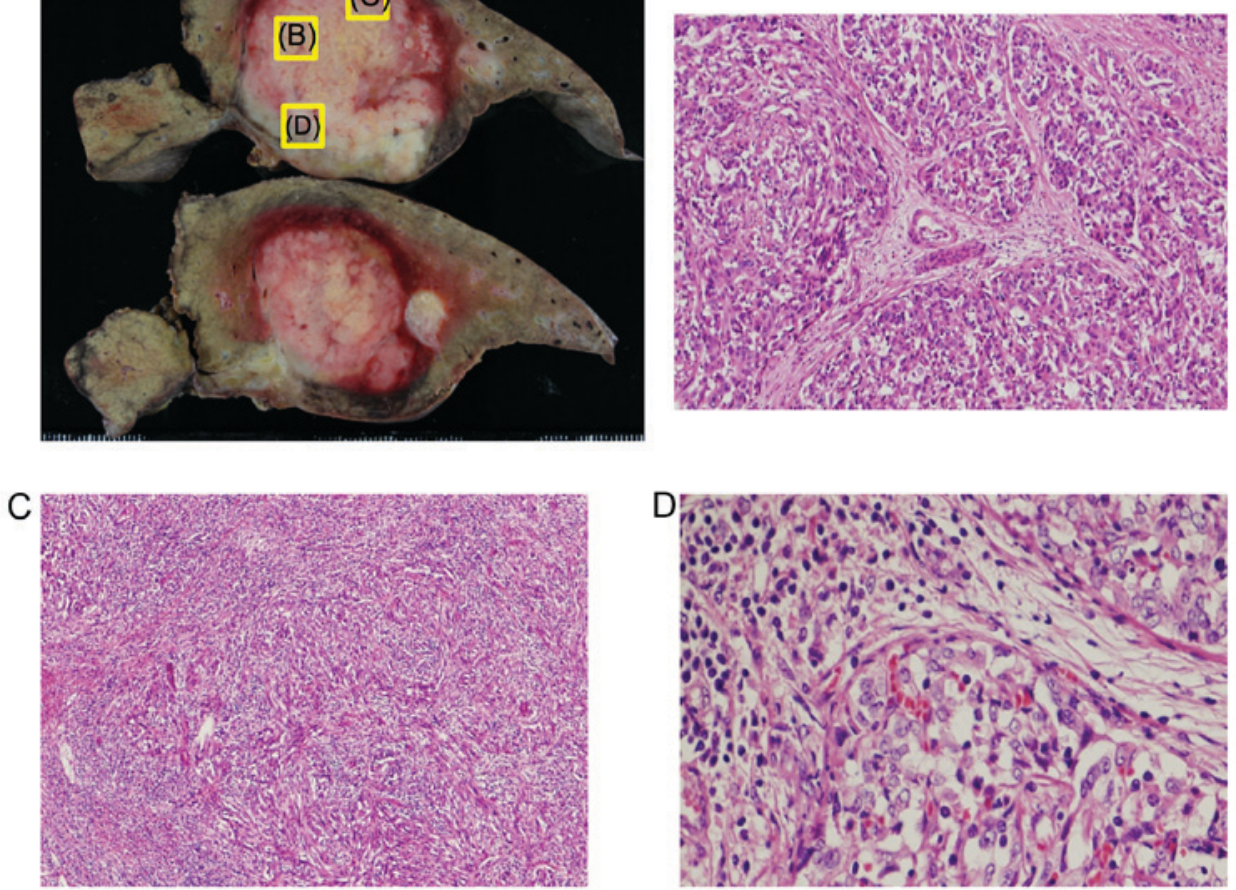

Figure 5. Macroscopic findings of resected liver tumor. (A) The axial cut surface of the resected specimen revealed a well-circumscribed whitish tumor, measuring 46x45x35 mm. Necrosis and bleeding were observed inside the tumor. The tumor exhibited three different histologies, including: (B) Polygonal eosinophilic carcinoma cells arranged in a cord- or nest-like pattern (magnification, x10); (C) spindle-shaped carcinoma cells with an irregular growth pattern; (magnification, x10) and (D) a number of cancer nests with irregular tubular structures (magnification, x20).

The resected liver tumor measured $46 \times 45 \times 35 \mathrm{~mm}$ (Fig. 5A). The tumor was histologically composed of three parts: i) Polygonal eosinophilic carcinoma cells arranged in a cord- or nest-like pattern; ii) spindle-shaped carcinoma cells with an irregular growth pattern; and iii) a number of cancer nests with irregular tubular structures (Fig. 5B-D). 
Table I. Comparison of histologic finding findings between needle biopsy specimen and surgical specimen.

\begin{tabular}{llll}
\hline $\begin{array}{l}\text { Special or } \\
\text { immunohistochemical staining }\end{array}$ & Needle biopsy specimen & Surgical specimen & Characteristics \\
\hline PAS and/or alcian-blue & Mucin production (-) & Mucin production (+) & ICC \\
EMA & Membranous positive & Membranous positive & \\
CK7 & Positive & Positive & \\
CK19 & Not determined & Positive & \\
CK20 & Negative & Negative & \\
CEA & Not determined & Negative & HCC \\
AFP & Negative & Negative & \\
HSA & Negative & Negative & Stem/progenitor cell \\
Glypican 3 & Positive & & \\
CD56 & Partially positive & Negative & \\
KIT & Negative & Negative &
\end{tabular}

ICC, intrahepatic cholangiocarcinoma; HCC, hepatocellular carcinoma; PAS, periodic acid-Schiff; EMA, epithelial membrane antigen; CK, cytokeratin; AFP, $\alpha$-fetoprotein; HSA, hepatocyte-specific antigen; CEA, carcinoembryonic antigen; CD56, cluster of differentiation 56.

A

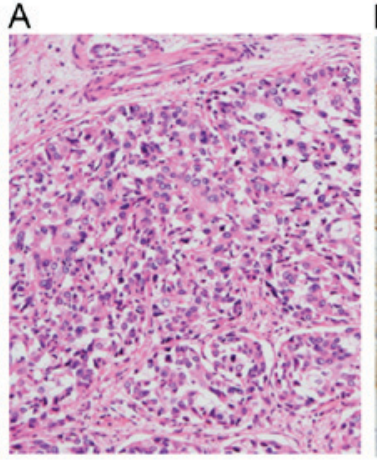

$\mathrm{E}$

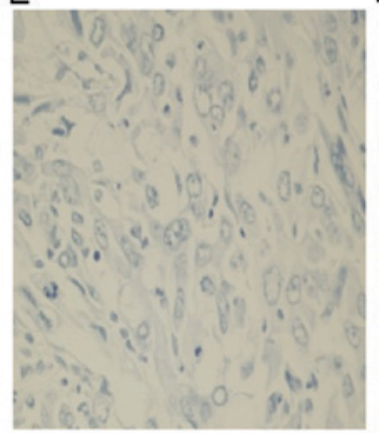

B

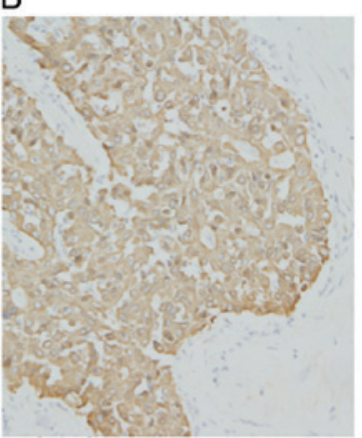

$\mathrm{F}$

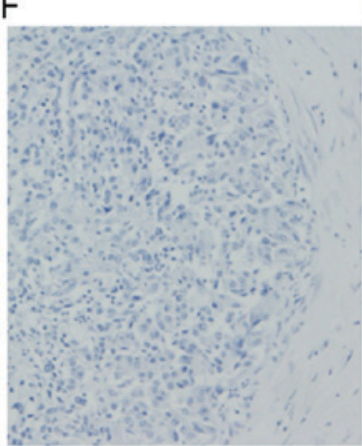

C

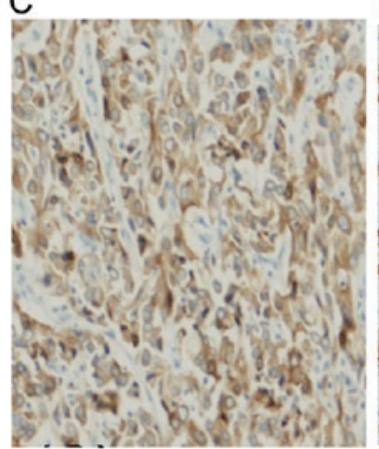

$\mathrm{G}$

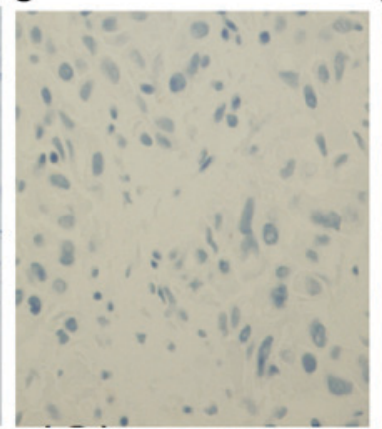

D

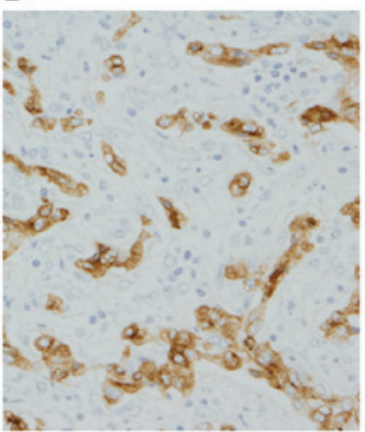

$\mathrm{H}$

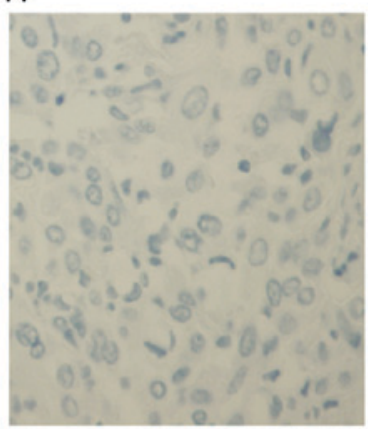

Figure 6. Histopathological analysis of resected liver tumor. (A) Hematoxylin-eosin staining demonstrated tumor cells with enlarged round nuclei and eosinophilic cytoplasm. Immunohistochemical staining of (B) CK7, (C) CK19, (D) EMA, (E) HSA, (F) glypican 3, (G) CD56 and (H) KIT demonstrated that the tumor cells were diffusely positive for CK7 and CK19, positive for EMA (in the luminal membranes), and negative for HSA, CD56 and KIT. HSA, hepatocyte-specific antigen; CD56, cluster of differentiation 56; CK, cytokeratin; EMA, epithelial membrane antigen (magnification, x20).

Additionally, mucin production was present in a portion of this tumor. This tumor did not exhibit histological features, and had admixtures of small monotonous glands, reflecting so-called antler-like anastomosing patterns as characteristics of $\mathrm{CoCC}$ (Fig. 6A). Immunohistochemical staining demonstrated that the tumor was positive for CKs 7, 19 (Fig. 6B and C) and EMA (Fig. 6D), and negative for AFP (Fig. 6E), hepatocyte-specific antigen, glypican 3, CD56 and KIT (Fig. 6F-H). EMA staining indicated that all of the tumor components in the resected specimen were cytoplasmic positive, but the normal liver was negative. This tumor did not exhibit characteristics of hepatic progenitor cells or stem cells. Based on these final histopathological and immunohistochemical findings, the tumor was diagnosed as a poorly differentiated ICC with anaplastic changes, not CoCC (Table I). The patient is still alive and has not suffered any recurrence at 15 months after surgery. 
Table II. Histopathological features of CoCC and ICC.

\begin{tabular}{lll}
\hline Histopathological features & \multicolumn{1}{c}{ CoCC } & \multicolumn{1}{c}{ ICC } \\
\hline Origin of tumor & $\begin{array}{l}\text { Cholangioles/ductules and the } \\
\text { canals of Hering } \\
\text { MMA immunohistochemical staining results }\end{array}$ & $\begin{array}{l}\text { Small intrahepatic bile ducts, } \\
\text { including interlobular bile ducts }\end{array}$ \\
Mucus production & Negative & Cytoplasmic positive \\
Classification according to the General & An independent disease entity & Positive
\end{tabular}

Rules for the Clinical and Pathological

Study of Primary Liver Cancer (9)

World Health Organization

classification 4th edition (21)

Subtype of CHC

ICC

CoCC, cholangiocellular carcinoma; ICC, intrahepatic cholangiocarcinoma; CHC, combined hepatocellular and cholangiocarcinoma; EMA, epithelial membrane antigen.

\section{Discussion}

ICC is the second most common type of primary liver cancer behind HCC in Japan in 2010 (1,11-16). A number of previous studies have reported rapidly rising rates of ICC over the recent decades globally $(13,17)$. ICC has similar risk factors to HCC, including cirrhosis of the liver, chronic viral hepatitis, alcohol abuse, diabetes mellitus and obesity (18). As ICC exhibits various radiological features, including capsular retraction, satellite nodules, and peripheral biliary dilatation, depending on the tumor's location, size, and intratumoral components $(3,19)$, a definitive pathological diagnosis is required for appropriate treatment planning. However, CoCC is a rare type of primary liver cancer (20). There are a number of reports regarding the definition of CoCC. Recently, CoCC was classified as a stem-cell subtype of combined hepatocellular and cholangiocarcinoma (CHC) according to the 2010 World Health Organization classification $(18,21)$. While, in Japan, CoCC was reclassified as a type of primary liver cancer according to he General Rules for the Clinical and Pathological Study of Primary Liver Cancer, which were developed by the Liver Cancer Study Group of Japan (22). Yano et al (23) reported that when the HCC or ICC area accounts for $\geq 10 \%$ of the tumor, the tumor should be diagnosed as $\mathrm{CHC}$. CoCC frequently exhibits HCC-like and ICC areas within the tumor, but these areas compose of $<10 \%$ of the tumor (24). Therefore, CoCC should be different from CHC. Histologically, ICC resembles small intrahepatic bile ducts, including interlobular bile ducts (13). Conversely, $\mathrm{CoCC}$ is considered to originate from the peripheral biliary duct system, including cholangioles/ductules and the canals of Hering, where hepatic progenitor cells are located (25). In a previous study, CoCC did not exhibit mucin production, although ICC did (25). Immunohistochemically, luminal EMA expression and positive CD56 are observed in CoCC, whereas cytoplasmic EMA expression and negative CD56 expression are usually observed in ICC (26). The histopathological features of these tumors are described in Table II. CoCC is rare primary malignant liver tumor, and therefore there are a limited number of reports regarding its prognosis $(20,27)$. The tumor doubling times of CoCC and ICC were reported to be 285 and 70 days, respectively (28). These data indicate that slow growth may be a characteristic of CoCC (28). Previous reports demonstrated CoCC primarily exhibited improved prognosis following resection, compared with ICC $(20,29)$.

In the present case, a preoperative diagnosis based on a combination of imaging and pathological information was subsequently determined to be incorrect. The characteristic imaging findings of $\mathrm{CoCC}$ include a combination of early peripheral enhancement, the presence of intratumoral portal tracts, the absence of bile duct dilation and delayed contrast enhancement (5). However, the characteristic imaging findings of ICC include peripheral rim-like enhancement, central delayed enhancement, irregular tumor margins and capsular retraction $(28,30)$. Therefore, the imaging findings of ICC are similar to those of CoCC. However, a number of hepatic tumors do not exhibit specific imaging findings. For example, the tumor in the present case exhibited HCC-like early enhancement in the arterial phase and ICC-like delayed enhancement in the portal and equilibrium phases; therefore, a percutaneous needle biopsy was performed. The biopsy specimen only included the part of the tumor containing spindle-shaped tumor cells with slightly eosinophilic cytoplasm and enlarged nuclei, which were arranged in a cord-like pattern. An immunohistochemical examination of the whole biopsy specimen demonstrated positive for $\mathrm{CK} 7$ and luminal EMA. However, the resected tumor revealed three distinct parts with different histological features, and an immunohistochemical examination indicated that the cytoplasm of the tumor cells was positive for CK7 and EMA. As was demonstrated in the present case, histopathological examinations of percutaneous needle biopsy samples do not always result in a correct diagnosis due to only small tumor samples being obtained (21). Intratumoral heterogeneity is increasingly recognized as a factor that has a major impact on diagnosis and the personalized treatment of a number of cancer types $(3,19)$. A previous study indicated that ICC exhibits substantial intratumoral heterogeneity, which should be considered when producing therapeutic decisions based on single biopsy 
examination $(3,19)$. Recently, numerous studies involving advanced technology have demonstrated that ICC can originate from multiple types of cells and exhibits heterogeneity in its genetic background and microenvironment $(3,19)$. The existence of two different stem cell compartments and the associated cell lineages may explain why ICC can develop from various types of cells (9). It has been indicated that ICC should be reclassified into ICC that originate from hepatic stem cell-derived lineages and ICC that originate from biliary tree stem/progenitor cell-derived lineages in the peribiliary glands or the epithelia of large bile ducts $(13,31)$. The genetic heterogeneity of ICC was examined in previous studies $(7,19)$, which indicated that the genetic variability in ICC could be caused by the complex interplay among a number of factors. A total of two genetically distinct stem cell niches exist along the biliary tree, and they exhibit differing susceptibility to various risk factors (19). Additionally, genetically distinct tumor cell sub-clones can co-exist with founder cells, which harbor the majority of the tumor-borne genetic mutations (19).

The microenvironmental factors associated with ICC include cancer-associated fibroblast and macrophage components (32). Crosstalk among different microenvironmental components is involved in the activation of ICC-associated oncogenic signaling (33). Additionally, an association between the imaging phenotypes and hypoxia-associated molecular profiles of ICC was demonstrated in a previous study (34).

The standard treatment strategy for cases of ICC involving a single intrahepatic nodule and no evidence of metastasis is surgical resection (18); however, patients that demonstrate extensive intrahepatic metastases/vascular invasion and/or notable regional lymph node metastasis should not undergo resection (35). Although the removal of the regional lymph nodes is recommended for its prognostic value (36), the present case involved notably swollen para-aortic lymph nodes. However, no appropriate treatment for $\mathrm{CoCC}$ has been established due to the rarity of the condition. Although Ariizumi et al (20) reported that patients with CoCC exhibited favorable long-term survival time following curative surgery, the effect of resection with curative intent in cases of CoCC involving possible metastasis to the regional lymph nodes is unknown. In the present case, if the ICC had been correctly diagnosed based on the preoperative biopsy examination, surgical resection would not have been performed. Considering the difficulty of differentiating between ICC and $\mathrm{CoCC}$, adopting a policy of resective surgery for these types of hepatic tumors is reasonable.

In conclusion, ICC is difficult to distinguish from other intrahepatic tumor types, including CoCC. Considering the difficulty of obtaining a definitive preoperative diagnosis, performing surgery as a diagnostic treatment may be reasonable in cases involving tumors that exhibit characteristics of ICC and other liver lesions without distant metastasis.

\section{Acknowledgements}

The authors would like to thank Professor Takehiro Otsubo (St. Marianna University School of Medicine), Professor Tomoaki Ichikawa (Saitama Medical University International Medical Center) and Professor Makoto Mochizuki (Teikyo
University), for the fruitful discussion regarding this patient at the 56th Liver Cancer Cases Conference.

\section{Funding}

No funding was received.

\section{Availability of data and materials}

The data used and/or analyzed during this published article are available from the corresponding author on reasonable request.

\section{Authors' contributions}

$\mathrm{KN}$, TE and JY collaborated in the conception and design of the study. KN, AK, MM, MN, MH, TN and SA acquired the data. KN, TE, HTa, SO, YK, ES, YY, SH, HTs, KH, HU and JY performed data analysis and interpretation. All authors were involved in writing the manuscript. All authors read and approved the final manuscript.

\section{Ethics approval and consent to participate}

Not applicable.

\section{Patient consent for publication}

The patient provided written informed consent for the publication of their data.

\section{Competing interests}

The authors declare that they have no competing interests.

\section{References}

1. Kitajima K, Shiba H, Nojiri T, Uwagawa T, Ishida Y, Ichiba N and Yanaga $\mathrm{K}$ : Intrahepatic cholangiocarcinoma mimicking hepatic inflammatory pseudotumor. J Gastrointest Surg 11: 398-402, 2007.

2. Adam SZ, Parthasarathy S and Miller FH: Intrahepatic cholangiocarcinomas mimicking other lesions. Abdom Imaging 40: 2345-2354, 2015.

3. Walter D, Döring C, Feldhahn M, Battke F, Hartmann S, Winkelmann R, Schneider M, Bankov K, Schnitzbauer A, Zeuzem S, et al: Intratumoral heterogeneity of intrahepatic cholangiocarcinoma. Oncotarget 8: 14957-14968, 2017.

4. Kozaka K, Matsui O, Kobayashi S, Koda W, Minami T, Kitao A, Inoue D, Yoneda N, Yoshida K, Toshima F, et al: Dynamic CT findings of cholangiolocellular carcinoma: Correlation with angiography-assisted CT and histopathology. Abdom Radiol (NY) 42: 861-869, 2017.

5. Tasch JJ and Dube N: An unusual presentation of advanced intrahepatic cholangiocarcinoma: When biopsy results fail. Am J Case Rep 19: 35-40, 2018.

6. Ishii N, Suzuki H, Tsukagoshi M, Watanabe A, Kubo N, Araki K, Wada S and Kuwano H: Giant cholangiolocellular carcinoma with early recurrence that was difficult to distinguish from cholangiocellular carcinoma: Report of a case. Int Surg 100: 1111-1116, 2015.

7. Bragazzi MC, Ridola L, Safarikia S, Matteo SD, Costantini D, Nevi L and Cardinale V: New insights into cholangiocarcinoma: Multiple stems and related cell lineages of origin. Ann Gastroenterol 31: 42-55, 2018.

8. Schlageter M, Terracciano LM, D'Angelo S and Sorrentino P: Histopathology of hepatocellular carcinoma. World J Gastroenterol 20: 15955-15964, 2014. 
9. Vijgen S, Terris B and Rubbia-Brandt L: Pathology of intrahepatic cholangiocarcinoma. Hepatobiliary Surg Nutr 6: 22-34, 2017.

10. Brierley JD, Gospodarowicz MK and Wittekind C (eds.): TNM Classification of Malignant Tumours. 8th edition. Wiley-Blackwell, Oxford, p272, 2017.

11. Nakanuma Y, Harada K, Ishikawa A, Zen Y and Sasaki M: Anatomic and molecular pathology of intrahepatic cholangiocarcinoma. J Hepatobiliary Pancreat Surg 10: 265-281, 2003.

12. Khan SA, Thomas HC, Davidson BR and Taylor-Robinson SD: Cholangiocarcinoma. Lancet 366: 1303-1314, 2005.

13. Nakanuma Y, Sato Y, Harada K, Sasaki M, Xu J and Ikeda H: Pathological classification of intrahepatic cholangiocarcinoma based on a new concept. World J Hepatol 2: 419-427, 2010.

14. Sakamoto Y, Kokudo N, Matsuyama Y, Sakamoto M, Izumi N, Kadoya M, Kaneko S, Ku Y, Kudo M, Takayama T, et al: Proposal of a new staging system for intrahepatic cholangiocarcinoma: Analysis of surgical patients from a nationwide survey of the Liver Cancer Study Group of Japan. Cancer 122: 61-70, 2016

15. Konstantinidis IT, Groot Koerkamp B, Do RK, Gönen M, Fong Y, Allen PJ, D'Angelica MI, Kingham TP, DeMatteo RP, Klimstra DS, et al: Unresectable intrahepatic cholangiocarcinoma: Systemic plus hepatic arterial infusion chemotherapy is associated with longer survival in comparison with systemic chemotherapy alone. Cancer 122: 758-765, 2016.

16. Buettner S, van Vugt JL, IJzermans JN and Groot Koerkamp B: Intrahepatic cholangiocarcinoma: Current perspectives. Onco Targets Ther 10: 1131-1142, 2017.

17. Malhi $\mathrm{H}$ and Gores GJ: Cholangiocarcinoma: Modern advances in understanding a deadly old disease. J Hepatol 45: 856-867, 2006.

18. Doherty B, Nambudiri VE and Palmer WC: Update on the diagnosis and treatment of cholangiocarcinoma. Curr Gastroenterol Rep 19: 2, 2017.

19. Brandi G, Farioli A, Astolfi A, Biasco G and Tavolari S: Genetic heterogeneity in cholangiocarcinoma: A major challenge for targeted therapies. Oncotarget 6: 14744-14753, 2015.

20. Ariizumi S, Kotera Y, Katagiri S, Nakano M, Nakanuma Y, Saito A and Yamamoto M: Long-term survival of patients with cholangiolocellular carcinoma after curative hepatectomy. Ann Surg Oncol 21 (Suppl 3): S451-S458, 2014.

21. Liu L, Wang W, Chen B, Xie W, Zhang Y, Zhang Z, Li Q, Li S, Hua Y, Shen S and Peng B: Combined hepatocellular and cholangiocarcinoma-WHO classi cation-based analysis of long-term prognosis after surgery. Int J Clin Exp Pathol 10: 6601-6612, 2017.

22. Kudo M, Kitano M, Sakurai T and Nishida N: General rules for the clinical and pathological study of primary liver cancer, nationwide follow-up survey and clinical practice guidelines: The outstanding achievements of the Liver Cancer Study Group of Japan. Dig Dis 33: 765-770, 2015.

23. Yano Y, Yamamoto J, Kosuge T, Sakamoto Y, Yamasaki S, Shimada K, Ojima H, Sakamoto M, Takayama T and Makuuchi M: Combined hepatocellular and cholangiocarcinoma-a clinicopathologic study of 26 resected cases. Jpn J Clin Oncol 33: 283-287, 2003.

24. Komuta M, Spee B, Vander Borght S, De Vos R, Verslype C, Aerts R, Yano H, Suzuki T, Matsuda M, Fujii H, et al: Clinicopathological study on cholangiolocellular carcinoma suggesting hepatic progenitor cell origin. Hepatology 47: $1544-1556,2008$
25. Yoh T, Kato T, Hirohata Y, Nakamura Y, Nakayama $\mathrm{H}$ and Okamura R: Cholangiolocellular carcinoma with rapid progression initially showing abnormally elevated serum alfa-fetoprotein. Clin J Gastroenterol 9: 257-260, 2016.

26. Hoshino H, Ohta M, Ito M, Uchimura K, Sakai Y, Uehara T, Low S, Fukushima M and Kobayashi M: Apical membrane expression of distinct sulfated glycans represents a novel marker of cholangiolocellular carcinoma. Lab Invest 96: 1246-1255, 2016.

27. Akabane S, Ban T, Kouriki S, Tanemura H, Nakazaki H, Nakano M and Shinozaki N: Successful surgical resection of ruptured cholangiolocellular carcinoma: A rare case of a primary hepatic tumor. World J Hepatol 9: 752-756, 2017.

28. Ishii N, Araki K, Yamanaka T, Handa T, Tsukagoshi M, Igarashi T, Watanabe A, Kubo N, Aishima S, Kuwano H and Shirabe K: Small cholangiolocellular carcinoma that was difficult to distinguish from cholangiocellular carcinoma: A case report. Surg Case Rep 3: 103, 2017.

29. Komuta M, Govaere O, Vandecaveye V, Akiba J, Van Steenbergen W, Verslype C, Laleman W, Pirenne J, Aerts R, Yano H, et al: Histological diversity in cholangiocellular carcinoma reflects the different cholangiocyte phenotypes. Hepatology 55: 1876-1888, 2012.

30. Motosugi U,Ichikawa T,Nakajima H,Araki T,MatsudaM,Suzuki T, Fujii H, Nakazawa T and Yamaguchi H: Cholangiolocellular carcinoma of the liver-imaging findings. J Comput Assist Tomogr 33: 682-688, 2009.

31. Cardinale V, Carpino G, Reid L, Gaudio E and Alvaro D: Multiple cells of origin in cholangiocarcinoma underlie biological, epidemiological and clinical heterogeneity. World J Gastrointest Oncol 4: 94-102, 2012.

32. Patel T: New insights into the molecular pathogenesis of intrahepatic cholangiocarcinoma. J Gastroenterol 49: 165-172, 2014.

33. Raggi C, Invernizzi P and Andersen JB: Impact of microenvironment and stem-like plasticity in cholangiocarcinoma: Molecular networks and biological concepts. J Hepatol 62: 198-207, 2015.

34. Sadot E, Simpson AL, Do RK, Gonen M, Shia J, Allen PJ, D'Angelica MI, DeMatteo RP, Kingham TP and Jarnagin WR: Cholangiocarcinoma: Correlation between molecular profiling and imaging phenotypes. PLoS One 10: e0132953, 2015.

35. Shimada K, Sano T, Nara S, Esaki M, Sakamoto Y, Kosuge T and Ojima $\mathrm{H}$ : Therapeutic value of lymph node dissection during hepatectomy in patients with intrahepatic cholangiocellular carcinoma with negative lymph node involvement. Surgery 145: 411-416, 2009.

36. Adachi T and Eguchi S: Lymph node dissection for intrahepatic cholangiocarcinoma: A critical review of the literature to date. J Hepatobiliary Pancreat Sci 21: 162-168, 2014.

This work is licensed under a Creative Commons Attribution-NonCommercial-NoDerivatives 4.0 International (CC BY-NC-ND 4.0) License. 